\title{
Generalized zero-one laws for large-order statistics
}

\section{HONG WANG}

Department of Mathematics and Statistics, Memorial University of Newfoundland, St John's, Newfoundland, Canada A1C 5S7.e-mail: hwang@morgan.ucs.mun.ca

For a fixed integer $r \geqslant 1$, let $Z_{r n}$ be the $r$ th largest of $\left\{X_{1}, X_{2}, \ldots, X_{n}\right\}$, where $X_{1}, X_{2}, \ldots$ is a sequence of i.i.d. random variables with the common distribution function $F(x)$. We prove that $P\left\{Z_{r n} \leqslant u_{n}\right.$, i.o. $\}=0$ or 1 accordingly as the series $\sum_{n=1}^{\infty} \exp \left[-n\left\{1-F\left(u_{n}\right)\right\}\right]\left[n\left\{1-F\left(u_{n}\right)\right\}\right]^{r} /$ $n<\infty$ or $=\infty$ for any real sequence $\left\{u_{n}\right\}$ such that $\lim _{n \rightarrow \infty} n\left\{1-F\left(u_{n}\right)\right\}=+\infty$. This weakens the condition added on the sequence $\left[n\left\{1-F\left(u_{n}\right)\right\}\right]$ by Wang and Tomkins and generalizes the results of Klass to the case when $r \geqslant 1$.

Keywords: i.i.d. random variables; large-order statistics; zero-one law

\section{Introduction}

Let $X_{1}, X_{2}, \ldots$ be a sequence of independent and identically distributed (i.i.d.) random variables with the common distribution function $F(x)$. For a fixed integer $r \geqslant 1$, let $Z_{r n}$ denote the $r$ th largest of $\left\{X_{1}, X_{2}, \ldots X_{n}\right\}$. Wang and Tomkins (1992) showed that, if $\left[n\left\{1-F\left(u_{n}\right)\right\}\right]$ is non-decreasing and divergent for a real sequence $\left\{u_{n}\right\}$, then the probability

$$
P\left\{Z_{r n} \leqslant u_{n} \text { i.o. }\right\}=0 \text { or } 1
$$

according to the convergence or divergence of any one of the following so-called criterion series:

$$
\begin{gathered}
\sum_{n=1}^{\infty} P\left\{Z_{r n} \leqslant u_{n}\right\}\left\{1-F\left(u_{n}\right)\right\} ; \\
\sum_{n=1}^{\infty} F^{n}\left(u_{n}\right) \frac{\left[n\left\{1-F\left(u_{n}\right)\right\}\right]^{r}}{n} ; \\
\sum_{n=1}^{\infty} \exp \left[-n\left\{1-F\left(u_{n}\right)\right\}\right] \frac{\left[n\left\{1-F\left(u_{n}\right)\right\}\right]^{r}}{n} ; \\
\sum_{n=1}^{\infty} \exp \left[-n\left\{1-F\left(u_{n}\right)\right\}\right] \frac{(\log \log n)^{r}}{n} ;
\end{gathered}
$$




$$
\sum_{n=3}^{\infty} F^{n}\left(u_{n}\right) \frac{(\log \log n)^{r}}{n} .
$$

The results of Wang and Tomkins (1992) generalized those of Klass $(1984 ; 1985)$ to the case $r \geqslant 1$ except for an additional monotonicity assumption which was added to the sequence $\left[n\left\{1-F\left(u_{n}\right)\right\}\right]$. From a counterexample (which will be published elsewhere), it is clear that the monotonicity condition added there is not extraneous. More precisely, the monotonicity of the sequence $\left[n\left\{1-F\left(u_{n}\right)\right\}\right]$ is essential for the series (1.5) and (1.6) to be criterion series. However, the series (1.2), (1.3) and (1.4) were seen to be valid criterion series in that counterexample, and this raises the following question: are any of the series in (1.2), (1.3) and (1.4) a criterion series for the probability (1.1) subject only to the hypothesis that $\left[n\left\{1-F\left(u_{n}\right)\right\}\right]$ is divergent? In this paper, we shall answer this question affirmatively for each of these three series.

To achieve these results, we shall modify the method of Klass (1984). The key difference between this method and that used by Wang and Tomkins (1992) relates to the choice of monitoring sequences. As observed by Klass (1984), for maximum effectiveness, such a monitoring sequence should relate to both the given distribution function $F(x)$ and the real sequence $\left\{u_{n}\right\}$. In this paper, we shall introduce several new monitoring sequences based on Klass's (1984) approach.

Klass (1984) showed that, for certain monitoring sequences $\left\{n_{k}\right\}$, the probability in (1.1) when $r=1$, will take values zero or one according as the series

$$
\sum_{k=1}^{\infty} P\left\{Z_{1 n_{k}} \leqslant u_{n_{k}}\right\}<\infty \text { or }=\infty .
$$

In this paper, we shall generalize this result to include the case where $Z_{1 n_{k}}$ is replaced by $Z_{r n_{k}}$, for a fixed integer $r \geqslant 1$. From the above, we shall prove our main results in Section 3, following the proof of a key lemma in Section 2. In Section 4 we shall present some remarks and elaboration on the main results.

\section{Two lemmas}

In this section, we shall present two lemmas which will play very important roles in the proof of the main results to be presented in the next section. The following lemma reduces to Lemma 1 of Klass (1984) when $r=1$ with a larger upper bound $C^{*}$.

Lemma 2.1. Let $X_{1}, X_{2}, \ldots$ be i.i.d. random variables and let $\left\{u_{n}\right\}$ be any non-decreasing real sequence. Fix an integer $k^{*}>1$ and let $n_{1}, n_{2}, \ldots, n_{k^{*}}$ be integers such that $0<n_{1} \leqslant n_{2} \leqslant \ldots \leqslant n_{k^{*}} \leqslant 2 n_{1}$. Let $P_{i}=P\left\{X_{1} \leqslant u_{n_{i}}\right\}, i \leqslant k^{*}$, and assume that $2 P_{1} \geqslant 1$, and $P_{i}^{n_{i}} \leqslant \mathrm{e}^{-1}, P_{i}^{n_{i+1}-n_{i}} \leqslant \lambda$, for all $1 \leqslant i \leqslant k^{*}$ and for some $0<\lambda<1$. Then there exists $a$ constant $C^{*}$, dependent only on $\lambda$ and $r$ such that 


$$
\sum_{i=1}^{k^{*}} P\left\{Z_{r n_{i}} \leqslant u_{n_{i}}\right\} \leqslant C^{*} \sum_{i=k_{*}}^{k^{*}} P\left\{Z_{r n_{i}} \leqslant u_{n_{i}}\right\},
$$

where $k_{*}$ is the smallest integer such that $P_{i} \geqslant P_{k^{*}}^{4}$ and $k_{*} \leqslant i \leqslant k^{*}$.

Proof. Let $\mathscr{D}_{j}=\left\{1 \leqslant k \leqslant k^{*}:\left[P_{k^{*}}\right]^{4 j}<P_{k} \leqslant\left[P_{k^{*}}\right]^{4^{j-1}}\right\}$. Note that $k^{*} \notin \mathscr{D}_{j}$ if $j>1$. Then let $m_{i}=-\log P_{i}$ and $\delta=\left(\log \lambda^{-1}\right)^{-1}$. Let $\hat{P}_{i}=P\left\{Z_{r n_{i}} \leqslant u_{n_{i}}\right\}$. Since the $\mathscr{D}_{j} \mathrm{~s}$ are disjoint,

$$
\sum_{i=1}^{k^{*}} \hat{P}_{i} \leqslant \sum_{j=1}^{\infty} \sum_{i \in \mathscr{D}_{j}} \hat{P}_{i}=\sum_{i \in \mathscr{D}_{1}} \hat{P}_{i}+\sum_{j=2}^{\infty} \sum_{i \in \mathscr{D}_{j}} \hat{P}_{i}
$$

Let $|A|$ and $I_{A}$ denote the cardinal number and the indicator function, respectively, of the set $A$. Then, for $j \geqslant 2$,

$$
\begin{aligned}
\left|\mathscr{D}_{j}\right|=\sum_{i=1}^{k^{*}} I_{\mathscr{D}_{j}}(i) & \leqslant \sum_{\left\{i<k^{*}: i \in \mathscr{D}_{j}\right\}}\left(n_{i+1}-n_{i}\right) m_{i} \delta \\
& \leqslant 4^{j} m_{k^{*}} \delta \sum_{\left\{i<k^{*}: i \in \mathscr{D}_{j}\right\}}\left(n_{i+1}-n_{i}\right) \\
& \leqslant 4^{j} m_{k^{*}} \delta\left(n_{k^{*}}-n_{1}\right) \\
& \leqslant 4^{j} n_{k^{*}} m_{k^{*}} \delta .
\end{aligned}
$$

Now, let $C_{r}^{*}=r 2^{r}$. By definition of $m_{i}$, we have $m_{k^{*}}=-\log P_{k^{*}} \geqslant 1-P_{k^{*}}$. We evaluate the second sum of (2.2) as follows:

$$
\begin{aligned}
\sum_{j=2}^{\infty} \sum_{i \in \mathscr{D}_{j}} P\left\{Z_{r n_{i}} \leqslant u_{n_{i}}\right\} & =\sum_{j=2}^{\infty} \sum_{i \in \mathscr{D}_{j}} P_{i}^{n_{i}} \sum_{t=0}^{r-1}\left(\begin{array}{c}
n_{i} \\
t
\end{array}\right)\left(\frac{1-P_{i}}{P_{i}}\right)^{t} \\
\left(\text { since } P_{i} \geqslant P_{1} \geqslant \frac{1}{2}\right) & \leqslant \sum_{j=2}^{\infty} \sum_{i \in \mathscr{D}_{j}} r 2^{r}\left\{n_{i}\left(1-P_{i}\right)\right\}^{r} P_{i}^{n_{i}} \\
& \leqslant \sum_{j=2}^{\infty}\left|\mathscr{D}_{j}\right| r 2^{r}\left\{n_{k^{*}}\left(1-P_{k^{*}}^{4^{j}}\right)\right\}^{r} P_{k^{*}}^{4^{j-1} n_{k^{*}} / 2} \\
& \leqslant \sum_{j=2}^{\infty}\left|\mathscr{D}_{j}\right| r 2^{r}\left[n_{k^{*}}\left\{\left(1-P_{k^{*}}\right)\left(\sum_{s=0}^{4^{j}-1} P_{k^{*}}^{s}\right)\right\}\right]^{r} P_{k^{*}}^{4^{j-1} n_{k^{*}} / 2}
\end{aligned}
$$




$$
\begin{aligned}
& \leqslant \sum_{j=2}^{\infty}\left|\mathscr{D}_{j}\right| r 2^{r}\left\{4^{j} n_{k^{*}}\left(1-P_{k^{*}}\right)\right\}^{r} P_{k^{*}}^{4^{j-1} n_{k^{*}} / 2} \\
& \leqslant r 2^{r}\left\{n_{k^{*}}\left(1-P_{k^{*}}\right)\right\}^{r} \sum_{j=2}^{\infty} 4^{j} \delta m_{k^{*}} n_{k^{*}} 4^{r j}\left\{\mathrm{e}^{-m_{k^{*}} n_{k^{*}}}\right\}^{2^{2 j-3}} \\
& \leqslant C_{r}^{*} \sum_{j=2}^{\infty} 4^{(r+1) j}\left(m_{k^{*}} n_{k^{*}}\right)^{r+1} \delta\left[\mathrm{e}^{-m_{k^{*}} n_{k^{*}}}\right]^{2^{2 j-3}} \\
& \leqslant C_{r}^{*} \sum_{j=2}^{\infty} 2^{2(r+1) j}\left(m_{k^{*}} n_{k^{*}}\right)^{r+1} \delta\left\{\mathrm{e}^{-m_{k^{*}} n_{k^{*}}}\right\}^{j} \\
& \leqslant C_{r}^{*}\left(m_{k^{*}} n_{k^{*}}\right)^{r+1} \delta\left\{1-2^{2(r+1)} \mathrm{e}^{-m_{k^{*}} n_{k^{*}}}\right\}^{-1}\left\{2^{4(r+1)} \mathrm{e}^{-2 m_{k^{*}} n_{k^{*}}}\right\} \\
& \leqslant 2 C_{r}^{*} 2^{4(r+1)}\left(m_{k^{*}} n_{k^{*}}\right)^{r+1} \delta \mathrm{e}^{-2 m_{k^{*}} n_{k^{*}}} \\
& \leqslant C_{r}^{*} 2^{4(r+2)} \delta\left\{\left(m_{k^{*}} n_{k^{*}}\right)^{r+1} \mathrm{e}^{-m_{k^{*}} n_{k^{*}}}\right\} \mathrm{e}^{-m_{k^{*}} n_{k^{*}}}
\end{aligned}
$$

(since $\left.\left(m_{k^{*}} n_{k^{*}}\right)^{r+1} \mathrm{e}^{-m_{k^{*}} n_{k^{*}}} \leqslant \mathrm{e}^{-1}\right) \leqslant C_{r}^{*} 2^{4(r+2)} \mathrm{e}^{-1} \delta P_{n_{k^{*}}}^{n_{k^{*}}}$

$$
\leqslant C_{r}^{*} 2^{4(r+2)} \mathrm{e}^{-1} \delta P\left\{Z_{r n_{k^{*}}} \leqslant u_{n_{k^{*}}}\right\} .
$$

Note that $\mathscr{D}_{1}=\left\{k_{*}, \ldots, k^{*}\right\}$; so $\sum_{i \in \mathscr{D}_{1}} \hat{P}_{i}=\sum_{i=k_{*}}^{k^{*}} \hat{P}_{i}$. Hence,

$$
\sum_{i=1}^{k^{*}} \hat{P}_{i} \leqslant\left(1+C_{r}^{*} 2^{4(r+2)} \mathrm{e}^{-1} \delta\right) \sum_{i=k_{*}}^{k^{*}} P\left\{Z_{r n_{i}} \leqslant u_{n_{i}}\right\} \equiv C^{*} \sum_{i=k_{*}}^{k^{*}} P\left\{Z_{r n_{i}} \leqslant u_{n_{i}}\right\} .
$$

This completes the proof.

The following lemma will be referred to frequently in the rest of the paper.

\section{Lemma 2.2.}

(i) For any $0 \leqslant z<1$,

$$
-\frac{z}{1-z} \leqslant \log (1-z) \leqslant-z
$$

(ii) For any $0 \leqslant z \leqslant \frac{1}{2}$ and $n \geqslant 1$,

$$
\exp \left\{-n\left(z+2 z^{2}\right)\right\} \leqslant(1-z)^{n} \leqslant \exp (-n z) \text {. }
$$

Proof. (i) and (ii) are easy consequences of Taylor expansion and Lemma 1.3.1 of Galambos (1987), respectively. 


\section{Generalized zero-one laws}

The following result is a key theorem in this paper which allows us to remove the monotonicity condition on the sequence $\left[n\left\{1-F\left(u_{n}\right)\right\}\right]$ for the criterion series (1.2), (1.3) and (1.4) used by Wang and Tomkins (1992). This result reduces to the key result of Klass (1984) when $r=1$.

Theorem 3.1. Let $X_{1}, X_{2}, \ldots$ be a sequence of i.i.d. random variables with common distribution function $F(x)$. Let $\left\{u_{n}\right\}$ be any non-decreasing real sequence such that

(i) $1-F\left(u_{n}\right) \rightarrow 0$ and

(ii) $n\left\{1-F\left(u_{n}\right)\right\} \rightarrow \infty$.

Fix an integer $r \geqslant 1$, take $r-1<\lambda_{*} \leqslant \lambda^{*}<\infty$, and choose any integers $1 \leqslant n_{1}<n_{2}<\ldots$ such that

$$
j\left\{1-F\left(u_{n_{k}}\right)\right\} \begin{cases}\geqslant \lambda_{*}, & \text { for } j \geqslant n_{k+1}-n_{k}, \\ \leqslant \lambda^{*}, & \text { for } j<n_{k+1}-n_{k} .\end{cases}
$$

Then

$$
P\left\{Z_{r n} \leqslant u_{n} \text { i.o. }\right\}=0 \text { or } 1
$$

according as the series

$$
\sum_{k=1}^{\infty} P\left\{Z_{r n_{k}} \leqslant u_{n_{k}}\right\}<\infty \text { or }=\infty
$$

Proof. Suppose that $\sum_{k=1}^{\infty} P\left\{Z_{r n_{k}} \leqslant u_{n_{k}}\right\}<\infty$, we have

$$
\begin{aligned}
P\left\{Z_{r n} \leqslant u_{n} \text { i.o. }\right\} & =\lim _{N \rightarrow \infty} P\left\{\bigcup_{k=N}^{\infty} \bigcup_{n_{k}<n \leqslant n_{k+1}}\left\{Z_{r n} \leqslant u_{n}\right\}\right\} \\
& \leqslant \lim _{N \rightarrow \infty} \sum_{k=N}^{\infty} P\left\{\bigcup_{n_{k}<n \leqslant n_{k+1}}\left\{Z_{r n} \leqslant u_{n}\right\}\right\} \\
& \leqslant \lim _{N \rightarrow \infty} \sum_{k=N}^{\infty} P\left\{Z_{r\left(n_{k}+1\right)} \leqslant u_{n_{k+1}}\right\} \\
& =\lim _{N \rightarrow \infty} \sum_{k=N}^{\infty}\left\{F\left(u_{n_{k+1}}\right)\right\}^{n_{k}+1} \sum_{j=0}^{r-1}\left(\begin{array}{c}
n_{k}+1 \\
j
\end{array}\right)\left(\frac{1-F\left(u_{n_{k+1}}\right)}{F\left(u_{n_{k+1}}\right)}\right)^{j} \\
& \leqslant \lim _{N \rightarrow \infty} \sum_{k=N}^{\infty} P\left\{Z_{r n_{k+1}} \leqslant u_{n_{k+1}}\right\} \frac{1}{\left\{F\left(u_{n_{k+1}}\right)\right\}^{n_{k+1}-n_{k}-1}}
\end{aligned}
$$




$$
\begin{aligned}
& \leqslant \lim _{N \rightarrow \infty} \sum_{k=N}^{\infty} P\left\{Z_{r n_{k+1}} \leqslant u_{n_{k+1}}\right\} \frac{1}{\left\{F\left(u_{n_{k}}\right)\right\}^{n_{k+1}-n_{k}-1}} \\
\text { (by Lemma 2.2) } & \leqslant \lim _{N \rightarrow \infty} \sum_{k=N}^{\infty} P\left\{Z_{r n_{k+1}} \leqslant u_{n_{k+1}}\right\} \mathrm{e}^{2\left(n_{k+1}-n_{k}-1\right)\left\{1-F\left(u_{n_{\mathrm{k}}}\right)\right\}} \\
(\text { by (3.1)) } & \leqslant \lim _{N \rightarrow \infty} \sum_{k=N}^{\infty} P\left\{Z_{r n_{k+1}} \leqslant u_{n_{k+1}}\right\} \mathrm{e}^{2 \lambda^{*}} \\
& =0 .
\end{aligned}
$$

Next, assume that $\sum_{k=1}^{\infty} P\left\{Z_{r n_{k}} \leqslant u_{n_{k}}\right\}=\infty$. Group the events $\left\{Z_{r n_{k}} \leqslant u_{n_{k}}\right\}$ into blocks as follows. Fix $0<\gamma<1$. Let $m_{0}=0$ and $m_{1}=n_{1}$, and, for $i \geqslant 1$,

$$
m_{i+1}=\min \left\{n_{k}>m_{i}: P\left\{Z_{1 m_{i}} \leqslant u_{n_{k}}\right\} \geqslant \gamma\right\} .
$$

Note that $m_{i+1}$ is always defined and finite since $P\left\{Z_{1 m_{i}} \leqslant u\right\}$ goes to 1 as $u$ tends to infinity.

Let $A_{i}=\bigcup_{m_{i} \leqslant n_{k}<m_{i+1}}\left\{Z_{r n_{k}} \leqslant u_{n_{k}}\right\}$ and $A_{i}^{\prime}=\bigcup_{m_{i} \leqslant n_{k}<m_{i+1}}\left\{Z_{r m_{i-1}, n_{k}} \leqslant u_{n_{k}}\right\}$, where $Z_{r m, n}$ is the $r$ th maxima of $\left\{X_{m+1}, X_{m+2}, \ldots, X_{n}\right\}$ when $n-m>r \geqslant 1$. For $j=0$ and $j=1$, the events $\left\{A_{2 i+j}^{\prime}: i \geqslant 1\right\}$ are independent. Applying the Borel-Cantelli lemma separately to even indices and odd indices, we see that, if

$$
\sum_{i=1}^{\infty} P\left(A_{i}^{\prime}\right)=\infty
$$

then $P\left\{A_{i}^{\prime}\right.$ i.o. $\}=1$. We claim that, in fact, (3.5) implies $P\left\{Z_{r n_{k}} \leqslant u_{n_{k}}\right.$ i.o. $\}=1$. To see this, suppose that (3.5) holds and fix $\varepsilon>0$. For each $i$, there exists an integer $c_{i}, i \leqslant c_{i}<\infty$, such that $P\left\{\bigcup_{j=i}^{c_{i}} A_{j}^{\prime}\right\}>1-\varepsilon$. Let

$$
\tau_{i}=\left\{\begin{array}{l}
\max \left\{j: A_{j}^{\prime} \text { occurs and } i \leqslant j \leqslant c_{i}\right\}, \\
\infty \text { if no such } j \text { exists. }
\end{array}\right.
$$

Note that $P\left\{\tau_{i}<\infty\right\}=P\left\{\bigcup_{j=i}^{c_{i}} A_{j}^{\prime}\right\}>1-\varepsilon$ and that $A_{j}^{\prime} \cap\left\{Z_{1 m_{j-1}} \leqslant u_{m_{j}}\right\} \subset A_{j}^{\prime} \cap$ $\left\{Z_{r m_{j}} \leqslant u_{m_{j}}\right\}$. Therefore,

$$
\begin{aligned}
P\left\{\bigcup_{j=i}^{c_{i}} A_{j}\right\} & \geqslant P\left\{\tau_{i}<\infty, A_{\tau_{i}}\right\} \\
& =\sum_{j=i}^{c_{i}} P\left\{\tau_{i}=j, A_{\tau_{i}}\right\} \\
& \geqslant \sum_{j=i}^{c_{i}} P\left\{\tau_{i}=j, Z_{r m_{j}} \leqslant u_{m_{j}}\right\}
\end{aligned}
$$




$$
\begin{aligned}
& \geqslant \sum_{j=i}^{c_{i}} P\left\{\tau_{i}=j, Z_{1 m_{j-1}} \leqslant u_{m_{j}}\right\} \\
& =\sum_{j=i}^{c_{i}} P\left\{\tau_{i}=j\right\} P\left\{Z_{1 m_{j-1}} \leqslant u_{m_{j}}\right\} \\
(\text { by }(3.4)) & \geqslant \gamma \sum_{j=i}^{c_{i}} P\left\{\tau_{i}=j\right\} \\
& \geqslant \gamma P\left\{\tau_{i}<\infty\right\} \\
& \geqslant \gamma(1-\varepsilon) .
\end{aligned}
$$

By the Hewitt-Savage zero-one law, we may conclude that $P\left\{Z_{r n_{k}} \leqslant u_{n_{k}}\right.$ i.o. $\}=1$. Since

$$
\sum_{i=1}^{\infty} P\left(A_{i}\right)=\infty
$$

implies (3.5), it is therefore sufficient to prove that the divergence of $\sum_{k=1}^{\infty} P\left\{Z_{r n_{k}} \leqslant u_{n_{k}}\right\}$ implies (3.7). To do so, we shall first find a lower bound for $P\left(A_{i}\right)$. To do this, we partition $A_{i}$ into sub-blocks of events, as follows. Fix $i$, let $m_{i, 1}=m_{i}$, and having defined $m_{i, 1}, m_{i, 2}$, $\ldots, m_{i, j}$, let

$$
m_{i, j+1}= \begin{cases}\min \left\{n_{k} \geqslant m_{i, j}+m_{i}\right\}, & \text { if such } n_{k} \leqslant m_{i+1} \text { exists } \\ m_{i+1}, & \text { otherwise. }\end{cases}
$$

Then set $\ell(i)=\max \left\{j: m_{i, j}<m_{i+1}\right\}$. For $1 \leqslant j<\ell(i)$, let

$$
A_{i, j}=\bigcup_{m_{i, j} \leqslant n_{k}<m_{i, j+1}}\left\{Z_{r n_{k}} \leqslant u_{n_{k}}\right\} .
$$

Thus $A_{i}=\bigcup_{j=1}^{\ell(i)} A_{i, j}$. Furthermore, for $j<\ell(i)$, define

$$
B_{i, j}=\left\{Z_{r m_{i, j+1}, m_{i, j+1}+m_{i}}>u_{i^{*}}\right\}
$$

where $i^{*}=\max \left\{n_{k}: n_{k}<m_{i+1}\right\}$.

Note that $A_{i, j} \cap B_{i, j}$, is disjoint from $A_{i, j^{\prime}}$ for $j^{\prime} \geqslant j+2$ and (3.4) ensures that $P\left\{Z_{1 m_{i}} \leqslant u_{i^{*}}\right\}<\gamma$. This allows us to place bounds on the probability $P\left\{Z_{r m_{i}} \leqslant u_{i^{*}}\right\}$, as follows:

$$
\begin{aligned}
\gamma>P\left\{Z_{1 m_{i}} \leqslant u_{i^{*}}\right\} & =\left[F\left(u_{i^{*}}\right)\right]^{m_{i}} \\
& =\exp \left\{m_{i} \log F\left(u_{i^{*}}\right)\right\} \\
\text { (by Lemma } 2.2(\mathrm{i})) & \geqslant \exp \left(-m_{i} \frac{1-F\left(u_{i^{*}}\right)}{F\left(u_{i^{*}}\right)}\right) \\
& \geqslant \exp \left\{-2 m_{i}\left[1-F\left(u_{i^{*}}\right)\right]\right\}
\end{aligned}
$$


if $i$ is large enough, i.e.,

$$
m_{i}\left\{1-F\left(u_{i^{*}}\right)\right\} \geqslant \log \left(\frac{1}{\gamma^{1 / 2}}\right) .
$$

Hence, with the fact that the function $x^{\alpha} \mathrm{e}^{-x} \downarrow$ in $[\alpha,+\infty)$, we can choose $\gamma$ so small that $\log \left(1 / \gamma^{1 / 2}\right) \geqslant r-1$ and, for each $i$,

$$
\begin{aligned}
r 2^{r}\left\{m_{i}\left\{1-F\left(u_{i^{*}}\right)\right\}\right]^{r-1} \exp \left[-m_{i}\left\{1-F\left(u_{i^{*}}\right)\right\}\right] & \leqslant r 2^{r}\left\{\log \left(\frac{1}{\gamma^{1 / 2}}\right)\right\}^{r-1} \cdot \gamma^{1 / 2} \\
& \equiv \gamma^{*}<1 .
\end{aligned}
$$

This yields

$$
\begin{aligned}
P\left(B_{i, j}\right) & =1-P\left\{Z_{r m_{i}} \leqslant u_{i^{*}}\right\} \\
& \geqslant 1-r 2^{r}\left[m_{i}\left\{1-F\left(u_{i^{*}}\right)\right\}\right]^{r-1} \exp \left[-m_{i}\left\{1-F\left(u_{i^{*}}\right)\right\}\right] \\
& >1-\gamma^{*}
\end{aligned}
$$

for $i$ large and all $1 \leqslant j \leqslant \ell(i)$. Thus, for such large $i$, we may use the simple inequality $2 P(A \cup B) \geqslant P(A)+P(B)$ to get

$$
\begin{aligned}
P\left(A_{i}\right) & \geqslant P\left\{\bigcup_{j=1}^{(i)}\left(A_{i, j} \cap B_{i, j}\right)\right\} \\
& \geqslant 2^{-1}\left[P\left\{\bigcup_{j \text { even }}\left(A_{i, j} \cap B_{i, j}\right)\right\}+P\left\{\bigcup_{j \text { odd }}\left(A_{i, j} \cap B_{i, j}\right)\right\}\right]
\end{aligned}
$$

(by disjointness) $=2^{-1} \sum_{j=1}^{\ell(i)} P\left(A_{i, j} \cap B_{i, j}\right)$

$$
\begin{aligned}
\text { (by independence) } & =2^{-1} \sum_{j=1}^{\ell(i)} P\left(A_{i, j}\right) P\left(B_{i, j}\right) \\
& >\left(1-\gamma^{*}\right) 2^{-1} \sum_{j=1}^{\ell(i)} P\left(A_{i, j}\right) .
\end{aligned}
$$

Fix $i \geqslant 1$, and $1 \leqslant j \leqslant \ell(i)$. Define

$$
k^{*}=\max \left\{k: n_{k}<m_{i, j+1}\right\}
$$

and

$$
k_{*}=\min \left\{k: n_{k} \geqslant m_{i, j}, P^{1 / 4}\left\{X \leqslant u_{n_{k}}\right\} \geqslant P\left\{X \leqslant u_{n_{k^{*}}}\right\}\right\} .
$$


For $m_{i, j} \leqslant n_{k}<m_{i, j+1}$, let

$$
B_{n_{k}}=\left\{Z_{r n_{k}, n_{k+1}}>u_{n_{k^{*}}}\right\}
$$

By the definition of $B_{n_{k}}$, it is easy to check that the events $\left\{Z_{r n_{k}} \leqslant u_{n_{k}}\right\} \cap B_{n_{k}}$, for $k_{*} \leqslant k \leqslant k^{*}$, are disjoint. Also, note that, for $k_{*} \leqslant k \leqslant k^{*}$,

$$
\begin{aligned}
P\left(B_{n_{k}}\right) & =1-P\left\{Z_{r n_{k}, n_{k+1}} \leqslant u_{n_{k^{*}}}\right\} \\
& =1-\left\{F\left(u_{n_{k^{*}}}\right)\right\}^{n_{k+1}-n_{k}} \sum_{j=0}^{r-1}\left(\begin{array}{c}
n_{k+1}-n_{k} \\
j
\end{array}\right)\left(\frac{1-F\left(u_{n_{k^{*}}}\right)}{F\left(u_{n_{k^{*}}}\right)}\right)^{j} \\
& \geqslant 1-\left\{F\left(u_{n_{k^{*}}}\right)\right\}^{n_{k+1}-n_{k}} \sum_{j=0}^{r-1}\left(\begin{array}{c}
n_{k+1}-n_{k} \\
j
\end{array}\right)\left(\frac{1-F\left(u_{n_{k}}\right)}{F\left(u_{n_{k}}\right)}\right)^{j} \\
& \geqslant 1-\left\{F\left(u_{n_{k}}\right)\right\}^{\left(n_{k+1}-n_{k}\right) / 4} r\left[\left(n_{k+1}-n_{k}\right)\left\{1-F\left(u_{n_{k}}\right)\right\}\right]^{r-1}
\end{aligned}
$$

(by Lemma 2.2) $\geqslant 1-r \exp \left(-\frac{n_{k+1}-n_{k}}{4}\left\{1-F\left(u_{n_{k}}\right)\right\}\right)\left[\left(n_{k+1}-n_{k}\right)\left\{1-F\left(u_{n_{k}}\right)\right\}\right]^{r-1}$

$$
\geqslant 1-r \lambda_{*}^{r} \exp \left(-\frac{\lambda_{*}}{4}\right) \equiv C_{*},
$$

using (3.1) and $x^{\alpha} \mathrm{e}^{-x} \downarrow$ in $[\alpha,+\infty)$ (since $\lambda_{*} \geqslant r-1$ ) in the last step. Thus,

$$
\begin{aligned}
P\left(A_{i, j}\right) & \geqslant P\left\{\bigcup_{k * \leqslant k \leqslant k^{*}}\left\{Z_{r n_{k}} \leqslant u_{n_{k}}\right\} \cap B_{n_{k}}\right\} \\
\text { (by disjointness) } & =\sum_{k_{*} \leqslant k \leqslant k^{*}} P\left\{\left\{Z_{r n_{k}} \leqslant u_{n_{k}}\right) \cap B_{n_{k}}\right\} \\
\text { (by independence) } & =\sum_{k_{*} \leqslant k \leqslant k^{*}} P\left\{Z_{r n_{k}} \leqslant u_{n_{k}}\right\} P\left(B_{n_{k}}\right) \\
& \geqslant C_{*} \sum_{k_{*} \leqslant k \leqslant k^{*}} P\left\{Z_{r n_{k}} \leqslant u_{n_{k}}\right\} \\
\text { (by Lemma 2.1) } & \geqslant \frac{C_{*}}{C^{*}} \sum_{m_{i, j} \leqslant n_{k} \leqslant m_{i, j+1}} P\left\{Z_{r n_{k}} \leqslant u_{n_{k}}\right\} .
\end{aligned}
$$

Set $S=\left\{(i, j): m_{i, j}\right.$ is defined $\}$. Then, from (3.9), 


$$
\begin{aligned}
\sum_{i=1}^{\infty} P\left(A_{i}\right) & \geqslant \frac{1-\gamma^{*}}{2} \sum_{i=1}^{\infty} \sum_{j=1}^{\ell(i)} P\left(A_{i, j}\right) \\
& =\frac{1-\gamma^{*}}{2} \sum_{\{(i, j) \in S\}} P\left(A_{i, j}\right) \\
& \geqslant \frac{\left(1-\gamma^{*}\right) C_{*}}{2 C^{*}} \sum_{k=r^{*}}^{\infty} P\left\{Z_{r n_{k}} \leqslant u_{n_{k}}\right\}=\infty
\end{aligned}
$$

for a positive integer $r^{*}$. This completes the proof.

To illustrate Theorem 3.1, we state the following theorem to get the full strength of what has actually been proved.

Theorem 3.2. Let $X_{1}, X_{2}, \ldots$ be a sequence of i.i.d. random variables with common distribution function $F(x)$. Let $\left\{u_{n}\right\}$ be any non-decreasing sequence satisfying (i) and (ii) of Theorem 3.1. Fix an integer $r \geqslant 1$, and take any $r-1<\lambda_{*} \leqslant \lambda^{*}<\infty$. Let $\left\{n_{k}\right\}$ be a nondecreasing sequence of positive integers. If, for all $k \geqslant 1$,

$$
\left(n_{k+1}-n_{k}\right)\left\{1-F\left(u_{n_{k}}\right)\right\} \geqslant \lambda_{*},
$$

then

$$
P\left\{Z_{r n_{k}} \leqslant u_{n_{k}} \text { i.o. }\right\}=1
$$

if and only if

$$
\sum_{k=1}^{\infty} \exp \left[-n_{k}\left\{1-F\left(u_{n_{k}}\right)\right\}\right]\left[n_{k}\left\{1-F\left(u_{n_{k}}\right)\right\}\right]^{r-1}=\infty .
$$

If, for all $k \geqslant 1$,

$$
\left(n_{k+1}-n_{k}\right)\left\{1-F\left(u_{n_{k}}\right)\right\} \leqslant \lambda^{*},
$$

then

$$
P\left\{Z_{r n_{k}} \leqslant u_{n_{k}} \text { i.o. }\right\}=0
$$

if and only if

$$
\sum_{k=1}^{\infty} \exp \left[-n_{k}\left\{1-F\left(u_{n_{k}}\right)\right\}\right]\left[n_{k}\left\{1-F\left(u_{n_{k}}\right)\right\}\right]^{r-1}<\infty
$$

Proof. By direct calculation, it is easy to get, for any fixed integer $r \geqslant 1$,

$$
\begin{aligned}
\frac{1}{(2 r)^{r}}\left\{F\left(u_{n_{k}}\right)\right\}^{n_{k}}\left[n_{k}\left\{1-F\left(u_{n_{k}}\right)\right\}\right]^{r-1} & \leqslant P\left\{Z_{r n_{k}} \leqslant u_{n_{k}}\right\} \\
& \leqslant r 2^{r}\left\{F\left(u_{n_{k}}\right)\right\}^{n_{k}}\left[n_{k}\left\{1-F\left(u_{n_{k}}\right)\right\}\right]^{r-1} .
\end{aligned}
$$


Thus, the series

$$
\sum_{k=r^{*}}^{\infty} P\left\{Z_{r n_{k}} \leqslant u_{n_{k}}\right\}
$$

and

$$
\sum_{k=r^{*}}^{\infty}\left[F\left(u_{n_{k}}\right)\right]^{n_{k}}\left[n_{k}\left\{1-F\left(u_{n_{k}}\right)\right\}\right]^{r-1}
$$

converge or diverge together.

By Lemma 2.2 (i), the convergence or divergence of both series (3.18) and

$$
\sum_{k=1}^{\infty} \exp \left[-n_{k}\left\{1-F\left(u_{n_{k}}\right)\right\}\right]\left[n_{k}\left\{1-F\left(u_{n_{k}}\right)\right\}\right]^{r-1}
$$

depends only on those terms for which $n_{k}\left\{1-F\left(u_{n_{k}}\right)\right\}<(1+\delta) \log k$, where $\delta$ is an arbitrary positive real number. For such terms $k$,

$$
\frac{\left\{F\left(u_{n_{k}}\right)\right\}^{n_{k}}}{\exp \left[-n_{k}\left\{1-F\left(u_{n_{k}}\right)\right\}\right]} \rightarrow 1 .
$$

In fact, if $n_{k}\left\{1-F\left(u_{n_{k}}\right)\right\} \geqslant(1+\delta) \log k$, then

$$
\exp \left[-n_{k}\left\{1-F\left(u_{n_{k}}\right)\right\}\right]\left[n_{k}\left\{1-F\left(u_{n_{k}}\right)\right\}\right]^{r-1} \leqslant \frac{\{(1+\delta) \log k\}^{r-1}}{k^{1+\delta}},
$$

since $x^{\alpha} \mathrm{e}^{-x} \downarrow$ in $[\alpha,+\infty)$. Thus, the series in (3.19) converges and, by Lemma 2.2 (i), so does the series in (3.17). Hence, the above two series converge and diverge together.

The next theorem, a generalization of the result of Klass (1985) in the case $r=1$, shows that the series (1.2), (1.3) and (1.4) are criterion series for (1.1), without any monotonicity assumption on the real sequence $\left[n\left\{1-F\left(u_{n}\right)\right\}\right]$.

Theorem 3.3. Let $X_{1}, X_{2}, \ldots$ be a sequence of i.i.d. random variables with common distribution function $F(x)$. Let $\left\{u_{n}\right\}$ be any non-decreasing real sequence satisfying (i) and (ii) of Theorem 3.1. Then

$$
P\left\{Z_{r n} \leqslant u_{n} \text { i.o. }\right\}=0 \text { or } 1
$$

according as

$$
\sum_{n=1}^{\infty} \exp \left[-n\left\{1-F\left(u_{n}\right)\right\}\right] \frac{\left[n\left\{1-F\left(u_{n}\right)\right\}\right]^{r}}{n}<\infty \text { or }=\infty .
$$

Moreover, the series in (3.22) can be replaced by (1.2) or (1.3).

Proof. Let $n_{1}=1$ and, having defined $n_{1}, n_{2}, \ldots, n_{k}$, let

$$
n_{k+1}=\min \left\{j>n_{k}:\left(j-n_{k}\right)\left\{1-F\left(u_{n_{k}}\right)\right\} \geqslant 1\right\} .
$$


Since $n\left\{1-F\left(u_{n}\right)\right\} \rightarrow \infty$ and $\left(n_{k+1}-n_{k}\right)\left\{1-F\left(u_{n_{k}}\right)\right\} \rightarrow 1$, it follows that $n_{k+1} / n_{k} \rightarrow 1$. Hence, there exists $k_{0}$ such that $n_{j}\left\{1-F\left(u_{n_{j+1}}\right)\right\} \geqslant r$, for $j \geqslant k_{0}$. Note that $y^{\alpha} \exp (-n y)$ decreases for $y \geqslant \alpha / n$. Thus, for all $k \geqslant k_{0}$,

$$
\begin{aligned}
& \sum_{n_{k} \leqslant n<n_{k+1}}\left\{1-F\left(u_{n}\right)\right\}^{r} n^{r-1} \exp \left[-n\left\{1-F\left(u_{n}\right)\right\}\right] \\
& \geqslant \sum_{n_{k} \leqslant n<n_{k+1}}\left\{1-F\left(u_{n_{k}}\right)\right\}^{r} n^{r-1} \exp \left[-n\left\{1-F\left(u_{n_{k}}\right)\right\}\right] \\
& \geqslant \sum_{n_{k} \leqslant n<n_{k+1}}\left\{1-F\left(u_{n_{k}}\right)\right\}^{r}\left(n_{k+1}-1\right)^{r-1} \exp \left[-\left(n_{k+1}-1\right)\left\{1-F\left(u_{n_{k}}\right)\right\}\right] \\
& \geqslant \sum_{n_{k} \leqslant n<n_{k+1}}\left\{1-F\left(u_{n_{k}}\right)\right\}^{r} n_{k}^{r-1} \exp \left[-n_{k}\left\{1-F\left(u_{n_{k}}\right)\right\}\right] \exp \left\{-\left(n_{k+1}-n_{k}-1\right)\left\{1-F\left(u_{n_{k}}\right)\right\}\right] \\
& \geqslant \mathrm{e}^{-1}\left(n_{k+1}-n_{k}\right)\left\{1-F\left(u_{n_{k}}\right)\right\}^{r} n_{k}^{r-1} \exp \left[-n_{k}\left\{1-F\left(u_{n_{k}}\right)\right\}\right] \\
& \quad \text { (by }(3.23)) \geqslant \mathrm{e}^{-1}\left[n_{k}\left\{1-F\left(u_{n_{k}}\right)\right\}\right]^{r-1} \exp \left[-n_{k}\left\{1-F\left(u_{n_{k}}\right)\right\}\right] .
\end{aligned}
$$

In the reverse direction, since $x \mathrm{e}^{x} \leqslant 2 \mathrm{e}^{2}$ if $0 \leqslant x \leqslant 2$,

$$
\begin{aligned}
& \sum_{n_{k} \leqslant n<n_{k+1}}\left\{1-F\left(u_{n}\right)\right\}^{r} n^{r-1} \exp \left[-n\left\{1-F\left(u_{n}\right)\right\}\right] \\
& \leqslant \sum_{n_{k} \leqslant n<n_{k+1}}\left\{1-F\left(u_{n_{k+1}}\right)\right\}^{r} n^{r-1} \exp \left[-n\left\{1-F\left(u_{n_{k+1}}\right)\right\}\right] \\
& \leqslant\left(n_{k+1}-n_{k}\right) \exp \left[-n_{k}\left\{1-F\left(u_{n_{k+1}}\right)\right\}\right]\left\{1-F\left(u_{n_{k+1}}\right)\right\}^{r-1} n_{k}^{r-1} \\
& \leqslant {\left[\left(n_{k+1}-n_{k}\right)\left\{1-F\left(u_{n_{k+1}}\right)\right\}\right] \exp \left[\left(n_{k+1}-n_{k}\right)\left\{1-F\left(u_{n_{k+1}}\right)\right\}\right] } \\
& \times\left\{1-F\left(u_{n_{k+1}}\right)\right\}^{r-1} n_{k+1}^{r-1} \exp \left[-n_{k+1}\left\{1-F\left(u_{n_{k+1}}\right)\right\}\right] \\
&= 2 \mathrm{e}^{2}\left[n_{k+1}\left\{1-F\left(u_{n_{k+1}}\right)\right\}\right]^{r-1} \exp \left[-n_{k+1}\left\{1-F\left(u_{n_{k+1}}\right)\right\}\right] .
\end{aligned}
$$

Hence, the series (1.4) and

$$
\sum_{k=1}^{\infty}\left[n_{k}\left\{1-F\left(u_{n_{k}}\right)\right\}\right]^{r-1} \exp \left[-n_{k}\left\{1-F\left(u_{n_{k}}\right)\right\}\right]
$$

converge or diverge together. Now the theorem follows from Theorem 3.1. The proof of the facts that (3.22) can be replaced by (1.2) and (1.3) can be found in the paper by Wang and Tomkins (1992). 


\section{Extensions of Theorem 3.1 and some remarks}

In this section, we shall make some remarks to conclude the paper.

Remark 4.1. The subsequence $\left\{n_{k}\right\}$ used in the proof of Theorem 2.1 of Wang and Tomkins (1992) was defined by

$$
n_{k}=\exp \left(\frac{\tau k}{\log k}\right), k=3,4, \ldots
$$

Note that this subsequence does not depend on either $\left\{u_{n}\right\}$ or the distribution function $F(x)$. While the use of this sequence led to a simpler argument because of its various nice analytical properties (Galambos 1987), its use required a monotonicity assumption on the sequence $\left[n\left\{1-F\left(u_{n}\right)\right\}\right]$. It is clear that Barndorff-Nielsen's (1961) method cannot be modified to produce Theorem 3.1, since the monotonicity of the sequence $\left[n\left\{1-F\left(u_{n}\right)\right\}\right]$ is essential to the proof of Lemma 1 of Barndorff-Nielsen (1961). However, note that in the proof of Theorem 3.1, the choice of $\left\{n_{k}\right\}$ involved both the real sequence $\left\{u_{n}\right\}$ and the distribution function $F(x)$, and a delicate refilling procedure was used to produce $m_{i}$ and $m_{i, j}$. This procedure ensured that we chose sufficient monitoring points to determine the pattern of occurrence of the events $\left\{Z_{r n} \leqslant u_{n}\right\}$; the subsequence defined by (4.1) is too sparse to do this job.

The next two remarks will present some other choices for the monitoring subsequences which can be used in place of that introduced in Theorem 3.1. These alternatives are analogous to those suggested by Klass (1984).

Remark 4.2. Choose $0<\lambda_{*} \leqslant \lambda^{*} \leqslant 1$ such that $2 r\left(\lambda^{*}\right)^{1 / 2}\left\{\log \left(1 / \lambda^{*}\right)\right\}^{r-1}<1$. Let $n_{1}=1$ and assume that, for $k>1, n_{k+1}$ satisfies

$$
P\left\{Z_{1 j} \leqslant u_{n_{k}}\right\} \begin{cases}\leqslant \lambda^{*}, & \text { for } j \geqslant n_{k+1}-n_{k}, \\ \geqslant \lambda_{*}, & \text { for } j<n_{k+1}-n_{k} .\end{cases}
$$

Then Theorem 3.1 remains true with this choice of $\left\{n_{k}\right\}$.

To see this, first assume that $\sum_{k=1}^{\infty} P\left\{Z_{r n_{k}} \leqslant u_{n_{k}}\right\}<\infty$. Then

$$
\begin{aligned}
P\left\{Z_{r n} \leqslant u_{n} \text { i.o. }\right\} & =\lim _{N \rightarrow \infty} P\left\{\bigcup_{k=N}^{\infty} \bigcup_{n_{k}<n \leqslant n_{k+1}}\left\{Z_{r n} \leqslant u_{n}\right)\right\} \\
& \leqslant \lim _{N \rightarrow \infty} \sum_{k=N}^{\infty} P\left\{\bigcup_{n_{k}<n \leqslant n_{k+1}}\left\{Z_{r n} \leqslant u_{n}\right\}\right\} \\
& \leqslant \lim _{N \rightarrow \infty} \sum_{k=N}^{\infty} P\left\{Z_{r\left(n_{k}+1\right)} \leqslant u_{n_{k+1}}\right\}
\end{aligned}
$$




$$
\begin{aligned}
& =\lim _{N \rightarrow \infty} \sum_{k=N}^{\infty} P\left\{Z_{r\left(n_{k}+1\right)} \leqslant u_{n_{k+1}}\right\} \frac{P\left\{Z_{1\left(n_{k}+1\right), n_{k+1}} \leqslant u_{n_{k+1}}\right\}}{P\left\{Z_{1\left(n_{k+1}-n_{k}-1\right)} \leqslant u_{n_{k+1}}\right\}} \\
& \leqslant \lim _{N \rightarrow \infty} \sum_{k=N}^{\infty} P\left\{Z_{r n_{k+1}} \leqslant u_{n_{k+1}}\right\}\left[P\left\{Z_{1\left(n_{k+1}-n_{k}-1\right)} \leqslant u_{n_{k+1}}\right\}\right]^{-1} \\
& \leqslant \lambda_{*}^{-1} \lim _{N \rightarrow \infty} \sum_{k=N}^{\infty} P\left\{Z_{r n_{k+1}} \leqslant u_{n_{k+1}}\right\} \\
& =0 .
\end{aligned}
$$

Secondly, assume that $\sum_{k=1}^{\infty} P\left\{Z_{r n_{k}} \leqslant u_{n_{k}}\right\}=\infty$. We need only re-evaluate the probability of $B_{n_{k}}$, as defined in the proof of Theorem 3.1, by noting that $\left[F\left(u_{n_{k}}\right)\right]^{n_{k+1}-n_{k}} \leqslant \lambda^{*}$ implies that

$$
-2\left(n_{k+1}-n_{k}\right)\left\{1-F\left(u_{n_{k}}\right)\right\} \leqslant-\left(n_{k+1}-n_{k}\right) \frac{1-F\left(u_{n_{k}}\right)}{F\left(u_{n_{k}}\right)}
$$

(by Lemma $2.2(\mathrm{i})) \leqslant\left(n_{k+1}-n_{k}\right) \log F\left(u_{n_{k}}\right)$

$$
\leqslant \log \lambda^{*}
$$

provided that $k$ is so large that $2 F\left(u_{n_{k}}\right)>1$. Thus,

$$
\begin{aligned}
P\left(B_{n_{k}}\right) & =1-P\left\{Z_{r\left(n_{k+1}-n_{k}\right)} \leqslant u_{n_{k^{*}}}\right\} \\
& \geqslant 1-r 2^{r} \exp \left[-\left(n_{k+1}-n_{k}\right)\left\{1-F\left(u_{n_{k}}\right)\right\}\right]\left[\left(n_{k+1}-n_{k}\right)\left\{1-F\left(u_{n_{k}}\right)\right\}\right]^{r-1} \\
& \geqslant 1-r 2^{r} \exp \left(-\frac{\log \left(\lambda^{*}\right)^{-1}}{2}\right)\left(\frac{\log \left(\lambda^{*}\right)^{-1}}{2}\right)^{r-1} \\
& =1-r 2^{r} \exp \left(\frac{\log \lambda^{*}}{2}\right)\left(\frac{\log \left(\lambda^{*}\right)^{-1}}{2}\right)^{r-1} \\
& =1-2 r\left(\lambda^{*}\right)^{1 / 2}\left\{\log \left(\frac{1}{\lambda^{*}}\right)\right\}^{r-1} \\
& >0 .
\end{aligned}
$$

The rest of the proof is exactly the same as the proof of Theorem 3.1.

Remark 4.3. Another choice of $\left\{n_{k}\right\}$ is given by the following construction. Choose $0<\lambda_{*} \leqslant \lambda^{*} \leqslant 1$. Let $n_{1}=1$ and, for $k>1$, define $n_{k+1}$ such that

$$
P\left\{Z_{1 j} \leqslant u_{n_{k}+j}\right\} \begin{cases}\leqslant \lambda^{*}, & \text { for } j=n_{k+1}-n_{k} \\ \geqslant \lambda_{*}, & \text { for } j<n_{k+1}-n_{k} .\end{cases}
$$


With this choice of $\left\{n_{k}\right\}$, Theorem 3.1 again remains true. To see this, suppose that the series $\sum_{k=1}^{\infty} P\left\{Z_{r n_{k}} \leqslant u_{n_{k}}\right\}<\infty$. Then, as before,

$$
\begin{aligned}
P\left\{Z_{r n} \leqslant u_{n}, \text { i.o. }\right\} & =\lim _{N \rightarrow \infty} P\left\{\bigcup_{k=N}^{\infty} \bigcup_{n_{k}<n \leqslant n_{k+1}}\left\{Z_{r n} \leqslant u_{n}\right\}\right\} \\
& \leqslant \lim _{N \rightarrow \infty} \sum_{k=N}^{\infty} P\left\{\bigcup_{n_{k}<n \leqslant n_{k+1}}\left\{Z_{r n} \leqslant u_{n}\right\}\right\} \\
& \leqslant \lim _{N \rightarrow \infty} \sum_{k=N}^{\infty} P\left\{Z_{r n_{k}} \leqslant u_{n_{k+1}}\right\} \\
& \leqslant \lim _{N \rightarrow \infty} \sum_{k=N}^{\infty} P\left\{Z_{r n_{k}} \leqslant u_{n_{k+1}}\right\} \frac{P\left\{Z_{1 n_{k}, n_{k+1}} \leqslant u_{n_{k+1}}\right\}}{\lambda *} \\
& \leqslant \lambda_{*}^{-1} \lim _{N \rightarrow \infty} \sum_{k=N}^{\infty} P\left\{Z_{r n_{k+1}} \leqslant u_{n_{k+1}}\right\} \\
& =0 .
\end{aligned}
$$

Finally, by the construction of the $n_{k}$ values,

$$
\begin{aligned}
-2\left(n_{k+1}-n_{k}\right)\left\{1-F\left(u_{n_{k}}\right)\right\} & \leqslant-2\left(n_{k+1}-n_{k}\right)\left\{1-F\left(u_{n_{k+1}}\right)\right\} \\
& \leqslant\left(n_{k+1}-n_{k}\right) \log F\left(u_{n_{k+1}}\right) \\
& \leqslant \log \lambda^{*} .
\end{aligned}
$$

In view of the approach used in the proof of Theorem 3.1 and Remark 4.2, it is now clear that the probability in (3.2) equals one if the series in (3.3) diverges with this choice of $n_{k}$.

\section{Acknowledgements}

The author is indebted to the referee for his/her comprehensive, insightful and helpful comments and suggestions which led to an improved presentation of the results.

This research was supported by Research Grant OGPIN-014, from the National Science and Engineering Research Council of Canada.

\section{References}

Barndorff-Nielsen, O. (1961) On the rate of growth of the partial maxima of a sequence of independent identically distributed random variables. Math. Scand., 9, 383-394. 
Galambos, J. (1987) The Asymptotic Theory of Extreme Order Statistics, 2nd edn. Malabar, FL: Robert E. Krieger.

Klass, M.J. (1984) The minimal growth rate of partial maxima. Ann. Probab., 12, 380-389.

Klass, M.J. (1985) The Robbins-Siegmund series criterion for partial maxima. Ann. Probab., 13, $1369-1370$.

Mori, T. (1976) Stability of sums of i.i.d. random variables when extreme terms are excluded from sums. Z. Wahrscheinlichkeitstheorie Verw. Geb., 36, 189-194.

Wang, H. and Tomkins, J. (1992) A zero-one law for large order statistics. Can. J. Statist., 20, 323334.

Received September 1994 and revised December 1996 